\title{
A STRONG TYPE $(2,2)$ ESTIMATE FOR A MAXIMAL OPERATOR ASSOCIATED TO THE SCHRÖDINGER EQUATION BY
}

\author{
CARLOS E. KENIG ${ }^{1}$ AND ALBERTO RUIZ
}

\begin{abstract}
Let $T^{*} f(x)=\sup _{t>0}\left|T_{t} f(x)\right|$, where $\left(T_{t} f\right)^{n}(\xi)=e^{i t|\xi|^{2}} \hat{f}(\xi) /|\xi|^{1 / 4}$. We show that, given any finite interval $I, \int_{I}\left|T^{*} f\right|^{2} d x \leqslant C_{I} \int_{\mathbf{R}}|f(x)|^{2} d x$, and that the above inequality is false with 2 replaced by any $p<2$. This maximal operator is related to solutions of the Schrödinger equation.
\end{abstract}

Introduction. Let $u(x, t)$ be the solution of the initial value problem $i \partial u(x, t) / \partial t$ $=\partial^{2} u(x, t) / \partial x^{2}$ in $t>0, \lim _{t \rightarrow 0} u(x, t)=f$, for $f$ say in $L^{2}(\mathbf{R})$, and where the limit is to be understood in the $L^{2}$-sense. By taking Fourier transforms, it is easy to see that $u(x, t)=S_{t} f(x)=\int_{-\infty}^{+\infty} e^{i x \xi} e^{i t \xi^{2}} \hat{f}(\xi) d \xi$. In [C], L. Carleson raised the question: Under what conditions does $u(x, t) \rightarrow_{t \rightarrow 0} f$ pointwise a.e.? Carleson showed in [C] that if $f$ belongs to the Sobolev space $H^{s}(\mathbf{R})=\left\{f \in L^{2}(\mathbf{R}): \int|\hat{f}(\xi)|^{2}\left(1+|\xi|^{2}\right)^{s} d \xi<\right.$ $\infty\}, s \geqslant 1 / 4, u(x, t) \rightarrow f$ a.e. as $t \rightarrow 0$. He also constructed an example of an $f \in H^{1 / 8}(\mathbf{R})$ such that $u(x, t)$ does not converge to $f$ a.e. In [DK], the authors showed that, in fact, given $s<1 / 4$, there exists $f \in H^{s}(\mathbf{R})$ so that $u(x, t)$ does not converge a.e. to $f$. Carleson obtained the positive result by showing (using the Kolmogorov-Seliverstov-Plessner method (see [KS])) that if $t(x)$ is an arbitrary measurable function of $x$, then the operator $S_{t(x)} f(x)=\int_{-\infty}^{+\infty} e^{i x \xi} e^{i t(x) \xi^{2}} \hat{f}(\xi) d \xi$ satisfies the inequality $\left|\int_{-1}^{1} S_{t(x)} f(x) d x\right| \leqslant C\|f\|_{H^{1 / 4}}$, for $f \in C_{0}^{\infty}$, with $C$ independent of $t(x)$ and $f$. This inequality is enough to guarantee almost everywhere convergence, but does not answer the question: What are the mapping properties of the maximal operator $S f=\sup _{0<t \leqslant 1}\left|S_{t} f(x)\right|$ ?

In this note we show that given any finite interval $I \subset \mathbf{R}$, we have the 'strong type' inequality $\int_{I}|S f|^{2} \leqslant C_{I}\|f\|_{H^{1 / 4}}^{2}$, where $C_{I}$ depends only on $I$. Moreover, the example in [DK] shows that the above inequality fails if $\|f\|_{H^{1 / 4}}$ is replaced by $\|f\|_{H^{s}}$ for any $s<1 / 4$. Instead of treating the operator $S$ for $f \in H^{1 / 4}$, we study the maximal operator $T f=\sup _{0<t \leqslant 1}\left|T_{t} f(x)\right|$, where $T_{t} f(x)=\int_{-\infty}^{+\infty} e^{i x \xi} e^{i t \xi^{2}} \hat{f}(\xi) d \xi /|\xi|^{1 / 4}$, and $f \in L^{2}(\mathbf{R})$ now, and we show that

$$
\int_{I}|T f|^{2} \leqslant C_{I}\|f\|_{L^{2}(\mathbf{R})}^{2}
$$

Moreover, the example in [DK] shows that the inequality

$$
\int_{I}|T f|^{p} \leqslant C_{I}\|f\|_{L^{p}(\mathbf{R})}^{p}
$$

Received by the editors March 15, 1982 and, in revised form, August 31, 1982.

1980 Mathematics Subject Classification. Primary 42A45; Secondary 42B25.

${ }^{1}$ Alfred P. Sloan Fellow 1981-83. Research supported in part by the NSF. 
is false for every $1 \leqslant p<2$. Thus, we encounter the curious phenomemon of a 'natural' maximal operator for which the interval of $p$ 's on which it is strongly bounded is not open. The operator, of course, involves cancellations. Examples of maximal operators with this property have been recently found in [NRS]. Their examples differ from ours in that they deal with positive operations, and therefore, cancellation is not an issue for them.

As in [C], our method of proof relies on the Kolmogorov-Seliverstov-Plessner ideas. We fix a function $t(x), 0<t(x)<1$, and consider the composition of $T_{t(x)}$ with its adjoint $T_{t(x)}^{*}$. By using asymptotic expansion and estimates for certain trigonometric integrals, we bound the $L^{2}$-norm of $T_{t(x)}\left(T_{t(x)}\right)^{*}$, independently of $t(x)$.

Finally, we would like to point out that many questions in this area remain unanswered. For example, we know that $T$ is unbounded on $L^{p}, p<2$, and bounded on $L^{2}$. Is $T$ bounded on $L^{p}, p>2$ ? This result should be true but is not obvious since $T$ is not a positive operation.

What are the analogues of our results for the Schrödinger equation in higher dimension?

What happens if, instead of the Schrödinger equation, we consider other (not necessarily constant coefficient) evolution equations of the same 'kind'?

Finally, we would like to point out that many of the integrals appearing in the sequel are not absolutely convergent, and should be interpreted suitably as 'oscillatory' or improper integrals. We have systematically ignored this point to avoid burdensome technical details.

Remark (AdDed IN PROOF). A. Nagel and E. M. Stein have recently given a simpler proof of the positive part of Theorem 2, using only the case $\xi_{0}=0$ of Proposition 1.

1. In this section we will establish our main result (Theorem 2). Our main tools are Propositions 1 and 2, which involve some (rather technical) estimates for trigonometric integrals. In order not to interrupt the flow of the proof of the main theorem, we postpone the proof of Propositions 1 and 2 until $\S 2$.

Proposition 1. For $a, b, \xi_{0} \in \mathbf{R}$, we have

$$
\left|\int_{-\infty}^{+\infty} e^{i\left(a \xi+h \xi^{2}\right)} \frac{d \xi}{|\xi|^{1 / 4}\left|\xi_{0}-\xi\right|^{1 / 4}}\right| \leqslant \frac{C}{|a|^{1 / 4}\left|2 b \xi_{0}+a\right|^{1 / 4}} .
$$

Proposition 2. For $a, b \in \mathbf{R}, 1 \geqslant b>0$,

$$
\int_{-\infty}^{+\infty} e^{i\left(a \xi+b \xi^{2}\right)} \frac{d \xi}{|\xi|^{1 / 4}}=\sqrt{\frac{\pi}{2}}(1+i) e^{-i\left(a^{2} / 4 b\right)} \cdot \frac{b^{-1 / 2}}{|a / 2 b|^{1 / 4}}+\Psi(a, b),
$$

where $|\Psi(a, b)| \leqslant C /|a|^{3 / 4}$, and $C$ is independent of $b$.

As in the Introduction, let $u(x, t)$ solve $i \partial u / \partial t-\partial^{2} u / \partial x^{2}=0$ in $(-\infty$, $+\infty) \times(0,+\infty)$ and satisfy $\lim _{t \rightarrow 0} u(x, t)=f(x)$, where the limit is taken in the $L^{2}$-sense. Then,

$$
u(x, t)=S_{t} f(x)=\int_{-\infty}^{+\infty} e^{i x \xi} e^{i t \xi} \hat{f}(\xi) d \xi
$$


Let $S f(x)=\sup _{0<t<1}\left|S_{t} f(x)\right|$, then

THEOREM 1. For any finite interval I,

$$
\int_{I}|S f|^{2} \leqslant C_{I}\|f\|_{H^{1 / 4}}^{2}
$$

where $C_{I}$ depends only on I. Moreover, if we replace $1 / 4$ in the previous inequality by any $s<1 / 4$, the inequality fails.

Let us now define

$$
T_{t} f(x)=\int_{-\infty}^{+\infty} e^{i x \xi} e^{i t \xi^{2}} \hat{f}(\xi) d \xi /|\xi|^{1 / 4}
$$

and $T f(x)=\sup _{0<t<1}\left|T_{t} f(x)\right|$ for $f \in L^{2}(\mathbf{R})$. Then

THEOREM 2. For any finite interval I,

$$
\int_{I}|T f|^{2} \leqslant C_{I} \int|f|^{2}
$$

where $C_{I}$ depends only on I. Moreover, if we replace 2 in the previous inequality by any $p<2$, the inequality fails.

The positive part of Theorem 1 follows directly from the positive part of Theorem 2. The negative part of Theorem 1 was established in [DK]. We therefore turn to the proof of Theorem 2 .

Proof of Theorem 2. Fix an interval $I$, and pick $0 \leqslant \varphi \in C_{0}^{\infty}(\mathbf{R}), \varphi \equiv 1$ on $I$. Let $t(x)$ be an arbitrary measurable function, $0<\varepsilon \leqslant t(x) \leqslant 1$. Consider the operator

$$
R f(x)=\varphi(x)=\int_{-\infty}^{+\infty} e^{i\left(x \xi+t(x) \xi^{2}\right)} \hat{f}(\xi) d \xi /|\xi|^{1 / 4} .
$$

The positive part of the theorem will follow immediately if we show that

$$
\int|R f|^{2} \leqslant C \int|f|^{2}
$$

where $C$ is independent of $t(x)$ and $\varepsilon$.

$$
R f(x)=\varphi(x) \int_{-\infty}^{+\infty} \int_{-\infty}^{+\infty} e^{i\left\{(x-y) \xi+t(x) \xi^{2}\right\}} f(y) d \xi /|\xi|^{1 / 4} d y
$$

where the double integral is interpreted in a suitable sense. Interchanging the order of integration,

$$
R f(x)=\varphi(x) \int_{-\infty}^{+\infty} f(y)\left\{\int_{-\infty}^{+\infty} e^{i\left\{(x-y) \xi+t(x) \xi^{2}\right\}} d \xi /|\xi|^{1 / 4}\right\} d y .
$$

We now apply Proposition 2 to the expression in brackets to obtain

$$
\begin{aligned}
R f(x)= & C \varphi(x) \int_{-\infty}^{+\infty} f(y) e^{-i\left((x-y)^{2} / 4 t(x)\right)} \cdot(t(x))^{-1 / 2} /|(x-y) / 2 t(x)|^{1 / 4} d y \\
& +\varphi(x) \int_{-\infty}^{+\infty} f(y) \Psi((x-y), t(x)) d y=V f(x)+U(x)
\end{aligned}
$$


But,

$$
|U(x)| \leqslant C \varphi(x) \int_{-\infty}^{+\infty} f(y) d y /|x-y|^{3 / 4},
$$

and so, since $\varphi$ is compactly supported, $\int|U|^{2} \leqslant C \int|f|^{2}$, by the classical theorem on fractional integration (see [S]). Thus, we are reduced to showing (*) with $R$ replaced by $V$. We will, in fact, show that the operator norm in $L^{2}$ of $V V^{*}$ (where $V^{*}$ is the $L^{2}$-adjoint of $V$ ) is bounded independently of $t(x)$ and $\varepsilon$. This clearly implies $(*)$ for $V$. We now proceed to compute $V^{*}$ and $V V^{*} f$. It is easy to see that

$$
V^{*} f(y)=C \int f(z) \varphi(z) e^{i(z-y)^{2} / 4 t(z)}(t(z))^{-1 / 2} /|(z-y) / 2 t(z)|^{1 / 4}
$$

and so

$$
\begin{aligned}
V V^{*} f(x)= & C \varphi(x) \int e^{-i(x-y)^{2} / 4 t(x)}(t(x))^{-1 / 2} /(|x-y| / 2 t(x))^{1 / 4} \\
& \cdot \int f(z) \varphi(z) e^{i(z-y)^{2} / 4 t(z)}(t(z))^{-1 / 2} /(|z-y| / 2 t(z))^{1 / 4} d z d y \\
= & C \varphi(x) \int f(z) \varphi(z)\left\{\int e^{i\left((z-y)^{2} / 4 t(z)-(x-y)^{2} / 4 t(x)\right\}}\right. \\
& \left.\cdot(t(x) t(z))^{-1 / 2} 1 /(|x-y||z-y| / 2 t(x) t(z))^{1 / 4} d y\right\} d z \\
= & C \varphi(x) \int f(z) \varphi(z) \frac{1}{(t(x) t(z))^{1 / 4}} \\
& \cdot\left\{\int e^{i\left\{(z-y)^{2} / 4 t(z)-(x-y)^{2} / 4 t(x)\right\}} \frac{1}{|x-y|^{1 / 4}|z-y|^{1 / 4}} d y\right\} d z \\
= & c \varphi(x) \int f(z) \varphi(z) \frac{1}{(t(x) t(z))^{1 / 4}} e^{i\left\{z^{2} / 4 t(z)-x^{2} / 4 t(x)\right\}} \\
& \cdot\left\{\int \exp i\left[y^{2}\left\{\frac{1}{4 t(z)}-\frac{1}{4 t(x)}\right\}+y\left\{\frac{2 x}{4 t(x)}-\frac{2 z}{4 t(z)}\right\}\right] \frac{1}{|x-y|^{1 / 4}} \frac{1}{|z-y|^{1 / 4}} d y\right\} d z .
\end{aligned}
$$

Now let

$$
\alpha=\frac{2 x}{4 t(x)}-\frac{2 z}{4 t(z)}, \quad \beta=\frac{1}{4 t(z)}-\frac{1}{4 t(x)} .
$$

We want to estimate

$$
\left|\int e^{i\left\{\alpha y+\beta y^{2}\right\}} \frac{d y}{|x-y|^{1 / 4}|z-y|^{1 / 4}}\right| .
$$

Set $\xi=y-x$. Then the integral becomes

$$
\left|\int e^{i\left\{(\alpha+2 \beta x) \xi+\beta \xi^{2}\right\}} \frac{d \xi}{|\xi|^{1 / 4}|(z-x)-\xi|^{1 / 4}}\right|
$$

which, by Proposition 1, is bounded by

$$
\frac{C}{|\alpha+2 \beta x|^{1 / 4}|2 \beta(z-x)+\alpha+2 \beta x|^{1 / 4}} .
$$


But, $\alpha+2 \beta x=(z-x) / 2 t(z)$ and $2 \beta(z-x)+\alpha+2 \beta x=(x-z) / 2 t(x)$, and hence, $\left|V V^{*} f(x)\right| \leqslant C \varphi(x) \int f(z) \varphi(z) /|x-z|^{1 / 2} d z$. But then, if we again invoke the theorem on fractional integration and the compact support of $\varphi$, we see that the operator norm of $V V^{*}$ is bounded independently of $t(x)$ and $\varepsilon$, and the positive part of Theorem 2 follows.

To establish the negative part of Theorem 2, pick $f \in C_{0}^{\infty}(\mathbf{R})$, even supported in $[-1,1]$. As in $[\mathbf{D K}]$, define $f_{t}(x)=f(x / t) e^{2 i x / t^{2}}$, and now define $h_{t}$ by $\hat{h}_{t}(\xi)=$ $|\xi|^{1 / 4} \hat{f}_{t}(\xi)$. For $x>0$, set $t(x)=t^{2} x$. The computation in [DK] shows that

$$
\left|T_{t(x)} h_{t}(x)\right|=\left|\frac{1}{\sqrt{x}} \int f(y) e^{i y^{2} / x} d y\right|=g(x),
$$

which is independent of $t$. Fix a $p<2$, and an interval $I$ (which we may assume is contained in $(0, \infty))$ and suppose that

$$
\left.\int_{I}\left|T f^{p} \leqslant C_{I} \int\right| f\right|^{p} \quad \text { for all } f \in L^{p}(\mathbf{R}) \text {. }
$$

Then,

$$
\int_{I}|g(x)|^{p}=\left.\int_{I}\left|T_{t(x)} h_{t}(x) p^{p} \leqslant \int_{I}\right| T h_{t}(x)\right|^{p} \leqslant C_{I} \int\left|h_{t}\right|^{p}
$$

We now proceed to estimate the $L^{p}$-norm of $h_{t}$. Note that the $L^{p}$ norm of $f_{t}$ is bounded by $t^{1 / p}$, while

$$
\left(f_{t}\right)^{\prime}=\frac{1}{t} f^{\prime}(x / t) e^{2 i x / t^{2}}+\frac{2 i}{t^{2}} f(x / t) e^{2 i x / t^{2}},
$$

and hence $\left\|\left(f_{t}\right)^{\prime}\right\|_{p} \leqslant C t^{1 / p-2}$ as $t \rightarrow 0$. An application of the three lines theorem to $\hat{h}_{t}(\xi)=|\xi|^{2} \hat{f}_{t}(\xi), \quad 0 \leqslant \operatorname{Re} z \leqslant 1$, shows that $\left\|h_{t}\right\|_{p} \leqslant C t^{1 / p(1-1 / 4)} t^{(1 / p-2) \cdot 1 / 4}=$ $C t^{(1 / p-1 / 2)}$. Therefore, $\int_{I}|g(x)|^{p} \leqslant C_{I} C t^{(1 / p-1 / 2)}$, which is a contradiction as $t \rightarrow 0$ since $g$ is not identically 0 . Theorem 2 is established.

2.

LEMMA 1. Let $f$ be a monotonic function, and consider the integral $I=$ $\int_{a}^{b} e^{i \varphi(x)} f(x) d x$. Then if (i) either $d \varphi / d x \geqslant \lambda$ or $d \varphi / d x \leqslant-\lambda, \lambda>0$, in $[a, b]$, and $d \varphi / d x$ is monotonic, $|I| \leqslant C \max _{[a, b]}|f(x)| \cdot 1 / \lambda$, and if (ii) $d^{2} \varphi / d x^{2} \geqslant \lambda$ or $d^{2} \varphi / d x^{2} \leqslant-\lambda, \lambda>0$, then $|I| \leqslant C \max _{[a, b]}|f(x)| \cdot 1 / \lambda^{1 / 2}$.

Proof. The proof follows immediately from the classical Van der Corput estimates (see [Z, Volume 1, p. 197]).

We will now use Lemma 1 in a standard way to estimate a trigonometric integral. Since the proof is routine but tedious, it will be omitted.

LEMMA 2. If $\beta<0$,

$$
\left|\int_{-\infty}^{+\infty} e^{i\left(2 A u+u^{2}\right)} \frac{d u}{|u|^{1 / 4}|\beta-u|^{1 / 4}}\right| \leqslant \frac{C}{|A|^{1 / 4}|\beta+A|^{1 / 4}} .
$$

We are now ready to prove Propositions 1 and 2 of $\S 1$. 
Proof of Proposition 1. First assume that $b \neq 0$. Then, without loss of generality we can assume $b>0$. Let $2 A=a b^{-1 / 2}$, and change variables, setting $u=b^{1 / 2} \varepsilon$. Our integral then becomes

$$
b^{-1 / 4} \int_{-\infty}^{+\infty} e^{i\left(2 A u+u^{2}\right)} \frac{d u}{|u|^{1 / 4}\left|\xi_{0} b^{1 / 2}-u\right|^{1 / 4}} .
$$

Obviously, we can assume $\xi_{0} b^{1 / 2} \leqslant 0$. Then, Lemma 2 gives the estimate

$$
\frac{C b^{-1 / 4}}{|A|^{1 / 4}\left|A+\xi_{0} b^{1 / 2}\right|^{1 / 4}}=\frac{C}{|a|^{1 / 4}\left|2 b \xi_{0}+a\right|^{1 / 4}},
$$

as desired.

If $b=0$, as the Fourier transform of $1 /|\xi|^{1 / 4}$ equals $C /|\xi|^{3 / 4}$ (see [S]), we have

$$
\int_{-\infty}^{+\infty} e^{i a \xi} \frac{d \xi}{|\xi|^{1 / 4}\left|\xi_{0}-\xi\right|^{1 / 4}}=C^{2} \int \frac{e^{-i \xi_{0} x}}{|x|^{3 / 4}} \frac{1}{|a-x|^{3 / 4}} d x
$$

and it is very easy to see that the absolute value of the second integral is dominated by $C /|a|^{1 / 2}$, as desired.

Proof of Proposition 2. We can assume $a>0$. Change variables to $t=b^{1 / 2} \xi$, and set $2 A=a b^{-1 / 2}$. The integral then becomes

$$
b^{-1 / 2+1 / 8} \int_{-\infty}^{+\infty} e^{i\left(2 A t+t^{2}\right)} \frac{d t}{|t|^{1 / 4}}=I(a, b) .
$$

Assume first that $0<A \leqslant 2$. Note then that $b^{-1 / 2} /|a / 2 b|^{1 / 4} \leqslant C / a^{3 / 4}$, and so in this case it is enough to show that

$$
b^{-1 / 2+1 / 8}\left|\int_{-\infty}^{+\infty} e^{i\left(2 A t+t^{2}\right)} \frac{d t}{|t|^{1 / 4}}\right| \leqslant \frac{C}{a^{3 / 4}} .
$$

Note that $b^{-1 / 2+1 / 8}=b^{-3 / 8} \leqslant C / a^{3 / 4}$, and so it is enough to show that the integral is bounded. To do so, split the integral into $\int_{-\infty}^{-1}+\int_{-1}^{1}+\int_{1}^{\infty}$. In the middle integral we use absolute value inside the integral, while in the two extreme ones we use the fact that $\left(d^{2} / d t^{2}\right)$ (phase) $=2$, and $1 /|t|^{1 / 4} \leqslant 1$, together with Lemma 1 .

Assume now that $A>2$, and split the integral into $\int_{-\infty}^{-2 A}+\int_{-2 A}^{-A / 2}+\int_{-A / 2}^{-1}+\int_{-1}^{1}$ $+\int_{1}^{\infty}$. In the first integral, $(d / d t)($ phase $)=2 A+2 t \leqslant-2 A$, in the third one, $2 A+2 t \geqslant A$, and in the last one $2 A+2 t \geqslant 2 A$, while in all of these integrals $1 /|t|^{1 / 4} \leqslant 1$. Hence, Lemma 1 shows that they are bounded by $C / A$. Hence, their contribution to $I(a, b)$ is bounded by

$$
\begin{aligned}
C \frac{b^{-3 / 8}}{A} & =\frac{C}{2} \frac{b^{-3 / 8}}{a} b^{1 / 2}=\frac{C}{2} \frac{b^{1 / 8}}{a}=\frac{C}{2} \frac{1}{a^{3 / 4}}\left(\frac{b^{1 / 2}}{a}\right)^{1 / 4} \\
& =C \frac{1}{a^{3 / 4}} \frac{1}{A} \leqslant \frac{C}{a^{3 / 4}} .
\end{aligned}
$$

Now,

$$
\left|\int_{-1}^{1} e^{i\left(2 A t+t^{2}\right)} \frac{d t}{|t|^{1 / 4}}\right|=\left|A^{-3 / 4} \int_{-A}^{A} e^{i\left(2 \xi+\xi^{2} / A^{2}\right)} \frac{d \xi}{|\xi|^{1 / 4}}\right|
$$


To study $\int_{-A}^{A}$, we split it into $\int_{-A}^{-1}+\int_{-1}^{1}+\int_{1}^{A}$. The middle one is bounded, by taking absolute value inside the integral. The two extremes ones are bounded since $1 /|\xi|^{1 / 4} \leqslant 1$, and $(d / d \xi)$ (phase) $=2+2 \xi / A^{2} \geqslant 1$ in both cases. Thus, the contribution of $\int_{-1}^{1}$ to $I(a, b)$ is bounded by $C b^{-3 / 8} / A^{3 / 4}=C / a^{3 / 4}$. It remains then to show that

$$
b^{-3 / 8} \cdot \int_{-2 A}^{-A / 2} e^{i\left(2 A t+t^{2}\right)} \frac{d t}{|t|^{1 / 4}}=(1+i) \sqrt{\frac{\pi}{2}} e^{-i\left(a^{2} / 2 b\right)} \frac{b^{-1 / 2}}{|a / 2 b|^{1 / 4}}+O\left(\frac{1}{|a|^{3 / 4}}\right)
$$

where $O$ is independent of $b$. By a change of variables $u=-t$,

$$
\int_{-2 A}^{-A / 2}=e^{-i A^{2}} \int_{A / 2}^{2 A} e^{i(t-A)^{2}} \frac{d t}{t^{1 / 4}}
$$

We now apply the mean value theorem to the function $1 / t^{1 / 4}$, near $t=A$. We thus obtain, for $A / 2 \leqslant t \leqslant 2 A$ (and remembering that $A>2$ ), $1 / t^{1 / 4}=1 / A^{1 / 4}-$ $(5 / 4)(t-A) /[A+\theta(t)]^{5 / 4}$, where $\theta(t)$ is an increasing function, with $|\theta(t)| \leqslant$ $|t-a|$. Hence, our integral becomes

$$
\frac{e^{-i A^{2}}}{A^{1 / 4}} \int_{-A / 2}^{A} e^{i \eta^{2}} d \eta-\frac{5}{4} e^{-i A^{2}} \int_{A / 2}^{2 A} e^{i(t-A)^{2}}(t-A) \frac{d t}{[A+\theta(t)]^{5 / 4}}=\mathrm{I}+\mathrm{II} .
$$

To estimate I, note that, as $\int_{-\infty}^{+\infty} e^{i \eta^{2}} d \eta=(1+i) \sqrt{\pi / 2}$,

$$
\int_{-A / 2}^{A} e^{i \eta^{2}} d \eta=(1+i) \sqrt{\frac{\pi}{2}}+O\left(\frac{1}{A}\right)
$$

so that

$$
\mathrm{I}=\frac{e^{-i A^{2}}}{A^{1 / 4}} \cdot(1+i) \sqrt{\frac{\pi}{2}}+O\left(\frac{1}{A^{5 / 4}}\right)
$$

Hence,

$$
b^{-3 / 8} \mathrm{I}=\frac{e^{-i a^{2} / 4 b}}{|a / 2 b|^{1 / 4}} b^{-1 / 2} \sqrt{\frac{\pi}{2}}(1+i)+b^{-3 / 8} O\left(\frac{1}{A^{5 / 4}}\right) .
$$

But, as $A>2, b^{-3 / 8} O\left(1 / A^{5 / 4}\right) \leqslant C / a^{3 / 4}$. So, all that remains to be shown is that $b^{-3 / 8}|\mathrm{II}| \leqslant C / a^{3 / 4}$. An integration by parts shows that

$$
|\mathrm{II}|=C\left|\frac{e^{i A^{2}}}{[A+\theta(2 A)]^{5 / 4}}-\frac{e^{i A^{2} / 4}}{[A+\theta(A / 2)]^{5 / 4}}-C \int_{A / 2}^{2 A} e^{i(t-A)^{2}} \frac{\theta^{\prime}(t)}{[A+\theta(t)]^{5 / 4+1}} d t\right| .
$$

Now, as $\theta$ is increasing, $|\theta(t)| \leqslant|t-A|$, we see that $|A+\theta(2 A)| \geqslant|A|$, while $|A+\theta(A / 2)| \geqslant A / 2$, so that the first two terms are bounded by $C / A^{5 / 4}$, which produces the required bound. The integral is bounded in absolute value by

$$
\begin{aligned}
C \int_{A / 2}^{2 A} \frac{\theta^{\prime}(t)}{[A+\theta(t)]^{5 / 4+1}} d t & \leqslant \frac{C}{A^{5 / 4+1}} \int_{A / 2}^{2 A} \theta^{\prime}(t) d t \\
& =\frac{C}{A^{5 / 4+1}} \theta(2 A)-\theta(A / 2) \leqslant \frac{C}{A^{5 / 4}},
\end{aligned}
$$

as desired. Hence, Proposition 2 is established. 


\section{REFERENCES}

[C] L. Carleson, Some analytical problems related to statistical mechanics, Euclidean Harmonic Analysis, Lecture Notes in Math., Vol. 779, Springer-Verlag, Berlin and New York, 1979, pp. 5-45.

[DK] B. E. J. Dahlberg and C. E. Kenig, A note on the almost everywhere behavior of solutions to the Schrödinger equation (Proc. of Italo-American Symposium in Harmonic Analysis, University of Minnesota), Lecture Notes in Math., Vol. 908, Springer-Verlag, Berlin and New York, 1982, pp. 205-208.

[KS] A. N. Kolmogorov and G. Seliverstov, Sur la convergence de séries de Fourier, C. R. Acad. Sci. Paris Sér. I Math. 178 (1925), 303-305.

[NRS] A. Nagel, W. Rudin and J. Shapiro, Tangential boundary behavior of function in Dirichlet type spaces, preprint.

[S] E. M. Stein, Singular integrals and differentiability properties of functions, Princeton Univ. Press, Princeton, N.J., 1971.

[Z] A. Zygmund, Trigonometric series, 2nd ed., Cambridge Univ. Press, London and New York, 1968.

School of Mathematics, University of Minnesota, Minneapolis, Minnesota 55455 